\title{
Las formas esquemáticas como contenido del cómic en la obra de Kevin Huizenga y Dan Zettwoch
}

\author{
Sergio Arredondo Garrido \\ Doctorando Dpto. Dibujo. Universidad de Granada \\ Profesor de Diseño Gráfico. Escuela de Arte de Granada
}

Sergio Arredondo Garrido (Granada, 1980) es licenciado en Bellas Artes por la Universidad de Granada, en la que actualmente es doctorando del Dpto. de Dibujo. Compagina su labor como investigador con la de profesor de Diseño Gráfico en la Escuela de Arte de Granada. Se ha dedicado a la ilustración, diseño gráfico y diseño tipográfico (granadatierrasoñada.es), recientemente su interés por el cómic y el diseño gráfico se ha puesto de manifiesto en las colaboraciones realizadas para el colectivo Ultrarradio (The Fall y Lagartija Nick "Hay Ruido Todavia"), así como en la publicación del cómic La Soga (Ultrarradio 2012), poniendo en práctica en estos los intereses de su investigación, los cuales versan sobre la relación entre las citadas disciplinas. 


\title{
Resumen
}

Este artículo pretende poner en valor la presencia del diseño de la información, expresado a través del lenguaje de la esquemática, como elemento que se integra en la narrativa del cómic.

Resulta posible encontrar elementos de este ámbito dentro de tipologías del cómic orientadas a la divulgación del conocimiento y, en los últimos años, estos recursos los encontramos integrados en obras de ficción, suponiendo una apuesta por las exploración de las posibilidades narrativas que ofrece el diseño en el cómic. En este artículo se pretende identificar formas de la esquemática que podemos localizar en la obra de Kevin Huizenga y Dan Zettwoch, así como sus capacidades expresivas.

\begin{abstract}
This article aims to highlight the presence of information design, expressed through schematics language, as an integrated element of the comic's narrative.

It is posible to find elements from this area inside some comic's typologies oriented to knowledge diffusion. Recently, we can also find these resources integrated to fiction works. They support the narrative possibilities that graphic design provide to comic. In this article we try to identify the schematics elements that we can find in the works of Kevin Huizenga and Dan Zettwoch.
\end{abstract}




\section{Presentación}

La historia del cómic es también la historia de sus exploraciones y de los consecuentes hallazgos narrativos. Es el resultado del establecimiento de unas convenciones que han hecho reconocible su sistema, su medio de difusión y su mercado. Del mismo modo que con facilidad podemos identificar qué es un cómic, la creatividad desarrollada por los autores implica un inmensa variabilidad de soluciones y revela transgresiones de las normas que se creían fijadas. No siempre la exploración de las fronteras es un objetivo en sí, sino que surge como una consecuencia de la búsqueda expresiva y narrativa más adecuada a las intenciones del autor, con la consecuente ampliación de las posibilidades expresivas del medio. La toma de conciencia de esta realidad para los creadores se ve refrendada en la opinión de Daniel Clowes, el cual afirmaba: "Sospecho que, incluso enfrentados a la más absoluta indiferencia, algunos de nosotros seguiremos creando cómics, aunque solo sea por la inmensa e inexplorada llanura que existe entre lo que se ha hecho y las emocionantes posibilidades que nos rodean en todas direcciones".

Este panorama de emocionantes posibilidades que nos confirma Clowes ha sido un incentivo para que la labor teórica y académica haya tratado al propio cómic como el soporte de su argumentación, ${ }^{2}$ demostrando su capacidad para servir de espacio al discurso teórico. En este marco, la conjunción de práctica y teoría del cómic ha pretendido aportar un análisis académico a la par que establecer con rigor cierta normalización del sistema del cómic. García Sánchez abordaba desde una perspectiva global al cómic, considerándolo como una forma inserta en el marco de la comunicación visual y exponiendo el contexto donde este se desarrolla profesionalmente. En este, encontramos una interesante idea que sirve de estímulo para nuestra aportación. Para el autor y profesor granadino el cómic "hoy en día sigue evolucionando hacia estructuras narrativas complejas en paralelo a la infografía" ${ }^{3}$ Esta referencia, entendiendo a la infografía como la expresión más convencional de la esquemática, revela la intención narrativa de esta forma de periodismo visual y la constancia que de ella tienen algunos autores. ${ }^{4}$ La cercanía de esta forma de comunicación al público generalista nos sugiere que el lector está mayoritariamente preparado para un territorio de exploración como el que nos ofrece el enriquecimiento del cómic a través de la esquemática.

Lejos de atender a esta observación, desde estos u otros estudios académicos no se ha profundizado en la investigación del lenguaje de la esquemática en el territorio del cómic. Esto no es óbice para que alguno de ellos se sirva de esquemas para hacer más inteligibles sus argumentos. Sucede al tratar de visualizar en un diagrama de superficies el espectro gráfico que conforma el "vocabulario de los cómics", 5 o bien,

\footnotetext{
${ }^{1}$ Clowes, D. “Modern Cartoonist”, en Eightball n. ${ }^{\circ}$ 18, inserto de 16 páginas. Seattle, Fantagraphics Books, 1997, p. 14, citado en García, S. La novela gráfica. Bilbao, Astiberri, 2010, p. 265.

2 De entre las cuales queremos destacar las del autor norteamericano Scott McCloud: Understanding Comics: The Invisible Art (1994), Reinventing Comics: How Imagination and Technology Are Revolutionizing an Art Form (2000) y Making Comics: Storytelling Secrets of Comics, Manga and Graphic Novels (2006); así como las del dibujante español Sergio García Sánchez: Sinfonia Gráfica: Variaciones en las unidades estructurales y narrativas del cómic (2000), Anatomía de una bistorieta (2004) y Cómo bacer un cómic (2009).

3 García Sánchez, S. Anatomía de una historieta. Madrid, Sinsentido, 2004, p. 9.

${ }^{4}$ García Sánchez pertenece al colectivo experimental Oubapo, donde destacaron Patrice Killoffer o Lewis Trondheim y, especialmente, Étienne Lécroart que resulta el actual impulsor del movimiento. Un repaso a las publicaciones OuPus n.os 1 , 2, 3 y 4 (L'Association, 1997, 2003, 2004 y 2005) del colectivo nos permite encontrar de manera frecuente experimentaciones narrativas y el paralelismo entre la obra de estos autores y ciertas prácticas del diseño de la información.
}

${ }^{5}$ McCloud, S. Understanding Comics: The Invisible Art. New York, Harper Perennial, 1994 pp. 27-28 
representar en diagrama de barras los resultados del "análisis de las transiciones". ${ }^{6}$ Sin duda estos recursos gráficos resultan vitales para apoyar visualmente su argumentación. Estos esquemas adquieren una importancia clave para dar credibilidad a la investigación, en la medida en que son gráficos que pretenden confirmar la veracidad de sus conclusiones. A pesar de lo cual las referencias a los esquemas como elementos integrantes del "vocabulario de los cómics" no existe. Resulta relevante como se ha rehuido a su investigación si tenemos en cuenta que el cómic de divulgación que, entre otros, han servido de estímulo para la obra de McCloud, ${ }^{7}$ Cartoon History of the Universe, nos ofrece algunas formas esquemáticas que han pasado desapercibidas para los estudiosos del cómic, tales como cronogramas, árboles evolutivos y cartografías. Tan solo se puede localizar una interesante y lúcida aproximación a este campo de estudio en el capítulo "Comics and the Grammar of Diagrams" (2010) del investigador Isaac Cates, dentro de la obra monográfica dedicada al dibujo como una forma de pensamiento en la obra de Chris Ware. Cates señala la amplitud de posibilidades que en apariencia presenta la diagramación y la querencia por los signos pictográficos en la obra del autor norteamericano. Aunque este artículo no se centre en la obra de este, resulta innegable que la importante influencia de Ware ha acrecentado el interés por investigar los territorios de yuxtaposición de la esquemática y el cómic.

\section{¿Qué es la esquemática y qué le ofrece al cómic como contenido?}

Los recursos gráficos que pretendemos poner en valor dentro del cómic pertenecen al amplio espectro de soluciones gráficas que operan bajo la ciencia de la esquemática. Esta se define como la "Parte de la ciencia de la comunicación visual que estudia el nuevo lenguaje gráfico de los esquemas como mensajes, y la esquematización como procedimiento para la visualización de conocimientos que no son visibles en la realidad.". ${ }^{8}$ Según esta interpretación estas formas gráficas responden a un proceso que se desarrolla en dos fases: la primera es una sucesión de observaciones y de abstracciones por medio de las cuales los datos, estados y fenómenos complejos son convertidos por el cerebro en formas mentales; la segunda parte es la plasmación visual de dichas formas mentales en esquemas, o sea, mensajes concretados mediante procedimientos gráficos. Esta definición no profundiza en sus formas visuales, sino que propone una metodología de creación y una finalidad, según la cual la esquemática presta atención a aquellos aspectos de la comunicación visual que ayudan a visualizar lo invisible, aquellas realidades que no tienen apariencia gráfica ya que no pertenecen al universo de la mímesis, es decir, de los iconos. Este sería, expresado de manera general, el aporte más significativo que como contenido supone la esquemática para el cómic, al ofrecerle los recursos gráficos para incorporar a la narración aspectos de la realidad no visibles.

La esquemática es originalmente un lenguaje visual que se considera propio de la comunicación gráfica de las esferas científica y técnica. Al menos es desde esta perspectiva desde la que fundamentalmente se ha estudiado. Allí el dibujo y el diseño sirven a la presentación de informaciones complejas, ayudando a su análisis y a la toma de decisiones. Permiten autonomía de lectura, modificación de la perspectiva de análisis y aprendizaje, por lo que los productos que genera la esquemática se consideran un "objeto de trabajo". 9 Estos instrumentos gráficos que surgen de la esquemática no tienen de por sí un valor narrativo, sino expositivo. Han de ser exploradas para encontrar la información en torno a los estados, las estructuras, las relaciones, los desarrollos temporales, las secuencias numéricas o los conceptos que se visualizan a través de patrones y relaciones reconocibles que solo pueden ser observadas mediante su representación gráfica. Estas formas esquemáticas tienden a ser abstractas, semánticas, complejas, mul-

\footnotetext{
${ }^{6}$ McCloud, S. Op. cit. pp. 74-80.

7 Así lo pone de manifiesto el propio McCloud en una entrevista concedida a la revista The Believer realizada por Chute Hillary. Se puede leer en http://www.believermag.com/issues/200704/?read=interview_mccloud (consultado 5 de octubre de 2014)

8 Costa, J. La esquemática: visualizar la información. Barcelona, Paidós, 1998, p. 112.

9 Bender, J. y Marrinan, M. The Culture of Diagram. Stanford, Calif., Stanford University Press, 2010.
} 
tidimensionales e informativas. ${ }^{10}$ En el contexto de la creación de cómic contemporánea encontramos que la integración de estos recursos y otros novedosos se hace cada vez más recurrente, pero dista del planteamiento que hemos descrito. Probablemente esto se deba a la labor que ha realizado la infografía en la propagación de estas formas de comunicación optando por soluciones más redundantes, estéticas, livianas, unidimensionales y familiares, ${ }^{11}$ lo que ha facilitado su aceptación popular. Su amplio ancho de banda comunicacional ha condicionado el ambiente del lector y del creador, que además están sumidos en una constante tecnificación cultural, donde la ciencia y los dispositivos tecnológicos invaden cualquiera de nuestras prácticas habituales. Estas realidades brevemente descritas implican una traslación de los códigos visuales desde las esferas técnica y científica a situaciones cotidianas, en la medida en que como ciudadanos estamos inmersos en un ecosistema visual de amplitud de nuevos registros gráficos. De alguna manera, desde el punto de vista del creador, este está inmerso en los ambientes que Barbieri describió como las "zonas intermedias entre dos ecosistemas diferentes, donde se puede jugar con los reglas de ambos”. ${ }^{12}$

Para poder establecer las formas gráficas que reconocemos en el cómic como transposición del lenguaje de la esquemática, se hace necesario establecer el espectro de soluciones gráficas y códigos visuales que trata de aglutinar este lenguaje para advertir el tipo de enriquecimiento que suponen, por lo que un acercamiento a las familias de esquemas puede aportar algo de luz a esta inmersión. Apoyándonos en Costa, ${ }^{13}$ aunque filtrando las familias que propone, simplificando su propuesta para apostar por destacar tan solo aquellas que se reconocen de manera fehaciente en el cómic.

Estas serían cuatro: a) La familia de esquemas diseñados para expresar estados de objetos o fenómenos: que comprenderían a todas las formas gráficas que no incorporen dimensión temporal tales como los mapas, las vistas de sección arquitectónica o los atlas anatómicos. Se caracterizan por exponer relaciones inalterables en su superficie y tienen un vínculo evidente con la representación de la realidad, aunque teñida de cierta complejidad al combinar vistas interiores y exteriores o puntos de vistas poco habituales. b) La familia de esquemas utilizados para expresar principalmente estructuras y relaciones: este grupo de esquemas está más asociado al término diagrama, en tanto que su rasgo común es el uso del vector de conexión, en la definición de la estructura y en las relaciones consecuentes de lo vínculos entre elementos. Estos pueden tomar la forma de diagrama de árbol, de organigrama empresarial, de diagramas de espinas o de redes de relaciones. Su naturaleza abstracta los hace ajenos a cualquier relación con el grupo anterior, aunque igualmente establezcan relaciones en su conjunto, estas son de carácter lineal y multidireccional, lo cual les permite converger o divergir. En algunos casos, la propia estructura de la relación determina el significado de esta, de manera que el organigrama además de visualizar relaciones evidencia jerarquía. c) E1 tercer grupo lo constituye la familia de los esquemas que sirven para visualizar datos: una rama de la esquemática en especial auge en el contexto actual. Su nivel de abstracción es el mayor de todos los expuestos, en tanto que posibilita asignar formas gráficas a colecciones de informaciones cuantificadas. Las formas habituales de estos son los propios de la estadística gráfica: gráficos de barras, gráficos de sectores, histogramas... sin embargo, la realidad de las propuestas nos of recen una habitual integración con otras familias de esquemas de manera que resulta habitual of recer datos vinculados, por ejemplo, a mapas en forma de atlas de datos. d) La familia de esquemas que expresan desarrollos en el tiempo: estos son los únicos que incorporan dimensión temporal y son ambivalentes puesto que los hay de apariencia abstracta e icónica. Podemos reconocerlos como cronogramas, diagramas de flujo o árboles genealógicos, o bien, cercanos al sistema del cómic como secuencias de instrucciones gráficas de procedimiento.

\footnotetext{
10 Costa, J. Op. cit. p.116.

11 Cairo, A. El alfabeto funcional. Madrid, Alamut, 2011, p.76.

12 Barbieri, D. Los lenguaje del cómic. Barcelona, Paidós, 1993, p.13.

13 Costa, J. Op. cit. pp.120-126.
} 
La realidad de la esquemática y su amplia aplicación en ámbitos dispares demuestra su capacidad de hibridación, lo que a menudo hace complicada la tarea de reducir a una sola familia su clasificación. Este hecho lo podemos constatar en la infografía periodística, la cual ha mostrado una especial habilidad para hacer cohabitar diferentes familias en una única noticia optimizando así el espacio dedicado a estas secciones en las páginas del diario y generando una singular riqueza comunicativa. ${ }^{14}$ Algunas de estas combinaciones planean sobre la ambiciosa idea de "escapar de la planicie", ${ }^{15}$ donde la búsqueda de una mayor densidad informativa permite a la esquemática expresarse a través de múltiples dimensiones de análisis: espacio, tiempo, datos, estructura y relaciones; exponiendo una ambiciosa representación poliédrica de la realidad, ofreciendo al lector la información para que, a través de su interrelación y exploración, construya su particular interpretación o, en caso de la infografía, la reconstrucción de la noticia. El desarrollo de las técnicas narrativas que con la esquemática se están poniendo a prueba desde el periodismo visual, nos ofrece un territorio de investigación donde encontrar técnicas y procedimientos aún pendiente de traslación al cómic. No será ahora el objeto de este artículo la esquemática del periodismo visual, no obstante, resulta inevitable pensar en él como el principal de los entornos enriquecedores para el cómic. El objetivo que nos ocupa es de carácter más global y pretende localizar las familias de esquemas en el cómic para señalar las posibles vías de enriquecimiento a través de una óptica general.

\section{Caso de estudio}

Como veníamos anunciando el título del artículo, el mercado editorial norteamericano es el que nos muestra los ejemplos más relevantes de esta permeabilidad al diseño de la información. La obra de Ware, acompañada de otros autores de menor relevancia internacional, tales como Kevin Huizenga y Dan Zettwoch, han hecho de esta impregnación una señal reconocible de sus piezas narrativas. Ware destaca por la difusión de sus obras y por haber acaparado gran parte de la investigación académica contemporánea, sin embargo, su obra es menos relevante en cuanto a la incorporación de esquemas de forma evidente, razón por la cual pretendemos orientar este estudio a sus coetáneos. Por su parte, la obra de Huizenga parece explicitar de manera evidente sus raíces en el diseño gráfico de la información ya que podemos encontrar una actitud consciente de exploración, en tanto que existen publicaciones concebidas bajo esta óptica de forma intencionada. Ejemplo de esto resulta Fight or Run: Shadow of the Chopper (2008) editado por Buenaventura Press, "Back Cover" publicado en Or Else n. ${ }^{\circ}$ 2, "Which Sentences are we diagramming?" en el n. 5 de OrElse (2008) ambas de la editorial canadiense Drawn \& Quarterly. Editorial donde también publicará el ambicioso cómic Gloriana (2012) que, aunque adquiere la apariencia convencional de cómic de ficción, dedica una considerable parte del mismo a experimentar con las posibilidades de la narración expositiva. Esta obra pone de manifiesto la integración de ficción y realidad para la divulgación, que resulta de la integración del cómic y la esquemática. Igualmente incorporó recursos esquemáticos en la serie Ganges publicada por Fantagraphics Books (número del 1 al 4 publicados entre 2006 y 2011), quizás su serie más laureada por la que ha recibido varios premios Ignatz. Por último, destacaremos uno de sus trabajos más singulares, un cómic de divulgación que combina hábilmente cultura popular y humor: Amazing Facts $\mathcal{E}$ Beyond! with Leon Beyond (2014). Este nace como un experimento para la web y recientemente ha sido editado por Uncivilized Books. La serie ha sido realizada de forma colaborativa con el también dibujante de cómics Ted May, y especialmente con Dan Zettwoch, autor al que prestaremos atención en este artículo, siendo Birdseye Bristoe (2012) su obra más reseñable, publicada también por Drawn And Quarterly, la cual se autoproclama How to book, por su particular inmersión en la narrativa de las instrucciones gráficas de los modos de empleo.

Centraremos nuestras observaciones en la obra de Kevin Huizenga y Dan Zettwoch por permitirnos justificar el proceso de inmersión y yuxtaposición de las disciplinas del cómic y el diseño gráfico. Además de ser relevantes por el amplio repertorio de recursos de la esquemática a través de los que se expresan en

\footnotetext{
${ }^{14}$ Franchi, F. “Literatura gráfica”, en VVAA. Malofiej 18. Pamplona, Index Book, 2011, pp. 50-57.

15 Tufte, E. Envisioning information. Cheshire, Conn., Graphics Press, 1995.
} 
sus obras, ambos profesionales alternan, o han alternado, el oficio entre creadores de cómic y diseñadores gráficos, por lo que de manera consciente y natural han trasladado el aprendizaje y las reflexiones teóricas a las posibilidades prácticas comunicativas de una a otra disciplina. A este respecto, K. Huizenga opinaba que es natural que un historietista esté interesado en el diseño gráfico:

Cuando diseñas una página, estás diseñando un mundo y estás haciendo mucho trabajo de diseñador como dibujante de cómic. Hay una cantidad enorme de habilidades que tienes que aprender, más allá de las habilidades para escribir o dibujar. También tienes que pensar como ponerlo todo junto, de manera que funcione junto como un paquete. ${ }^{16}$

La realidad de esta interdisciplinariedad se debe a la pertenencia de ambos al equipo de diseñadores y dibujantes de la empresa Xplane: the visual thinking company, afincada en St. Louis (EE.UU.), donde eran responsables de la creación de visual explanations bajo demanda y para otras compañías, o bien, de sus particulares Xplanations que servían como proyectos de autopromoción. Las publicaciones realizadas carecen de autoría evidente, al estar realizadas bajo el paraguas corporativo y estético de Xplane, pero señalan estrategias narrativas cercanas a la yuxtaposición del cómic y el diseño gráfico. Kevin Huizenga dejó la empresa en 2001, mientras que Dan Zettwoch, aunque siguió sus pasos, ha seguido colaborando con la misma como freelance. ${ }^{17}$

Hemos tratado de situar al lector frente a un caso de estudio que cumpla con el requisito de permeabilidad evidente entre disciplinas, donde existan publicaciones profesionales de editoriales de prestigio y dentro de las cuales hayan publicado obras de ficción y no ficción. Satisfechas estas circunstancias podremos localizar las formas que pertenecen al lenguaje de la esquemática en un ámbito ajeno de aquel que nos ha servido para definir el lenguaje. Para poder comprender las posibilidades narrativas de estos casos de estudio, hemos pretendido establecer brevemente cual es la condición de este lenguaje, así como hacer una propuesta taxonómica de las familias que lo componen. De esta manera podremos profundizar en los recursos explorados y mostrar el enriquecimiento implícito en su uso. Para organizar el análisis de las piezas tomaremos como sistema rector la misma clasificación de familias realizada, ejemplificando en todos los casos con una o más piezas de los citados autores e incluyendo las referencias online para poder consultar los mismos.

\section{a) Estados}

El caso del mapa es sin duda el recurso esquemático más habitual y el paradigma del esquema de estados. Resulta destacable la rápida incorporación del mismo al cómic debido a que podemos encontrarlo incluido en la edición de Voyages et aventures du Dr. Festus (1840) de Rodolphe Töpffer. Este mapa se dibujó $^{18}$ para poder ayudar a entender los viajes del Doctor Festus, para explorar un espacio de ficción donde recrear las andanzas del protagonista. La incorporación de mapas a los cómics les proporciona, ante todo, verosimilitud, al establecer una relación espacial coherente y creíble donde desarrollar el relato, especialmente indicado para las historias de ficción. También el narrador, como el cartógrafo, determina las fronteras del espacio que será construido, elige qué elementos serán incluidos, establece la escala, los hitos, y así sucesivamente, dado que narrar es esencialmente una forma de trazar mapas, de orientarse y orientar a los lectores en un espacio concreto.

\footnotetext{
${ }^{16}$ Froenlich, C. "Gotham City and Metropolis can keep their clichés- St. Louis is flying high in the edgy world of alternative comics", entrevista con Kevin Huizenga, en St. Louis Magazine. 2007. http://www.stlmag.com/Cartoon-Heroes/ (consultado el 8 de mayo de 2013).

${ }_{17}$ Algunos ejemplos de las evidentes influencias se pueden observar en el blog de D. Zettwoch: $\underline{\text { http://zettwoch.blogspot. }}$ com.es/2008/03/gross-anatomy.html (consultado el 10 de Junio de 2014).

${ }^{18}$ Tal y como se recoge en una leyenda integrada en el mismo no fue creado por el autor del cómic sino por Jean Renaud.
} 


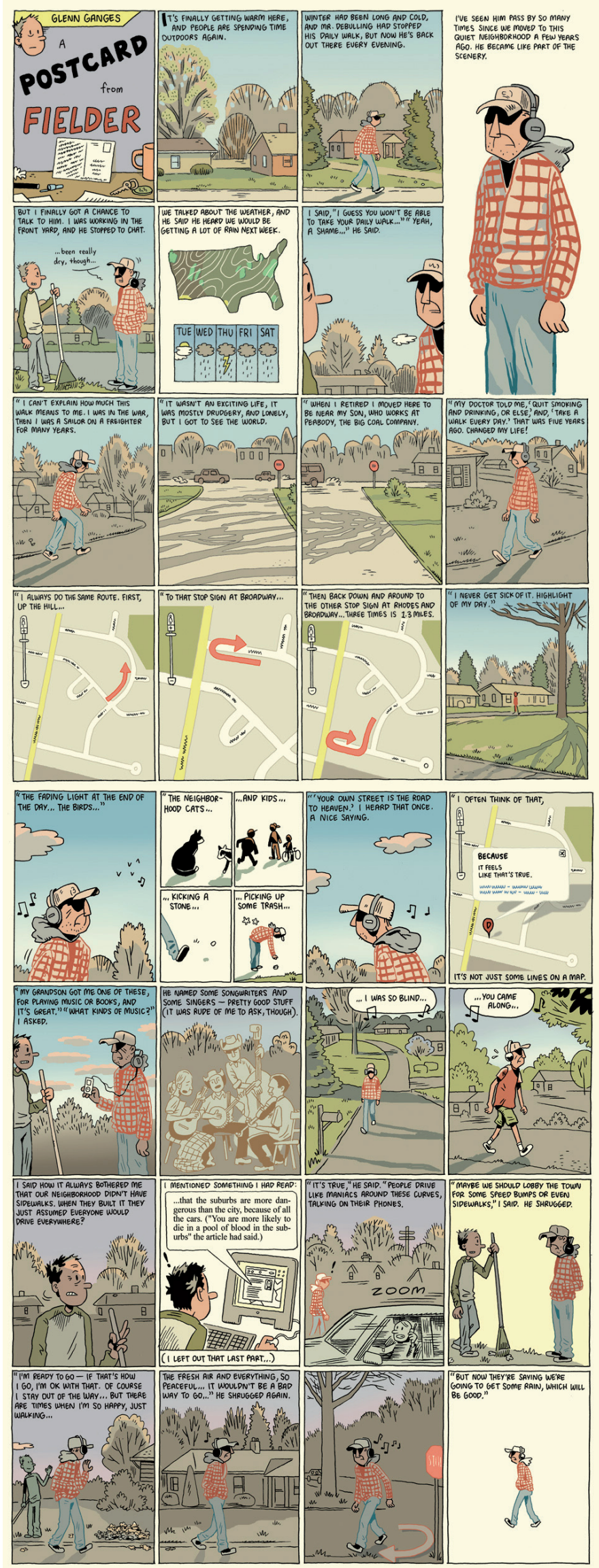

FIG. 1. A postcart from fielder. K. Huizenga. 27 de Julio de 2011. http://whatthingsdo.com/comic/a-postcard-from-fielder/ 
Glenn Ganges, el personaje más habitual de entre los creados por el autor, describe en esta postal a un personaje del vecindario, un paseante habitual al que ha observado rutinariamente en los momentos dedicados a sus labores de jardinero. En una charla con él, este individuo le explica su recorrido habitual por los suburbios de la ciudad. Para que el lector pueda visualizar la ruta por el vecindario, Huizenga se apoya en un mapa que repite en diferentes viñetas para poder incidir en la secuencia de los momentos y movimientos claves que determinan su ruta. Dado que la relación tecnológica con el mapa se ha modificado actualmente, el autor traslada este cambio del aspecto acercándolo al patrón gráfico que se ha hecho habitual por su eficacia, el de aplicaciones web, especialmente el de GoogleMaps. Es tal la apropiación estética que de la plataforma digital se hace para la secuencia del paseo, que colores y formas gráficas aluden a esta, hasta el punto de abstraer al personaje como si se tratara del símbolo de ubicación, y al verbalizar su opinión el vecino, esta se identifica con la ventana flotante que se despliega al hacer clic sobre el símbolo de ubicación. Los esquemas para expresar estados de objetos y fenómenos, se prestan en este ejemplo a ser secuenciados, para hacer en tres viñetas una descripción de espacio y movimiento, sin mención aparente a más temporalidad que la de la ordenación de los movimientos del paseante. Espacio, tiempo y acción se presentan codificados a través de combinación la cartografía, secuencia y vectores. Una estrategia narrativa que incide sobre el lugar y el itinerario antes que sobre el individuo.

\section{B) ESTRUCTURAS Y RELACIONES}

Este apartado es menos reconocible en los cómic de ficción. En ocasiones hemos encontrado alusiones a organigramas y, de manera extraordinaria, relaciones entre individuos, objetos y atributos como sucede en el n. 11 de Hawkeye: "Pizza is my bussines" de D. Aja y M. Fraction (2012). En cualquier caso no se encuentran muchos ejemplos donde la propia diagramación de los sistemas esquemáticos de estructuras y relaciones sean los protagonistas de la historia a excepción de los experimentos de Huizenga y Zettwoch.

La pieza que presentamos (FIG. 2) es el relato acerca de las situaciones donde varias citas textuales de artículos abordan de manera constante al lector. La única manera de visualizar el obsesivo análisis que de las frases hace el autor es evidenciándolo a través de su esquematización tras cada una de las citas. Para representar esta actitud se utiliza un diagrama de espinas de pez, que evoluciona a través de las viñetas, hacia diferentes relaciones y conexiones esquemáticas entre conceptos hasta adquirir algunos apariencia icónica, y finalmente un reclamo para que el lector complete y comparta la inquietud del autor. En esta pieza el protagonista fundamental es el texto y las relaciones entre los conceptos y las frases. Esta tipología de esquema de estructura y relaciones, ha sido utilizada por Kellogg-Reed ${ }^{19}$ en ámbito de la gramática para la representación de las componentes de una frase. Una fórmula esquemática que está presente en algunas aulas obedeciendo a criterios pedagógicos para la enseñanza de la gramática.

Esta singular propuesta invita a que sea el texto el elemento secuenciador y protagonista en la gran mayoría de las viñetas, aun así incorpora un alter ego de sí mismo, accionando el constante ejercicio de análisis y, posteriormente, incorpora la figura de uno de los personajes a los que hace referencia la cita, como protagonista final del cuestionamiento que se le realiza al lector.

Este diagrama de relaciones lo hemos seleccionado para establecer una paradoja que se da como fruto de la hibridación de familias de esquemas e intenciones narrativas. Se trata de un diagrama que simplifica y presenta de manera sencilla las relaciones existentes entre los miembros de la familia Greasepaühlts. Una familia irreal que resulta de las asociaciones inventadas de numerosos protagonistas de series televisivas $\mathrm{u}$ obras clásicas de la literatura. Este esquema, a medio camino entre el diagrama de árbol y árbol genealógico, explicita las relaciones conyugales, así como la procreación o la pertenencia —en referencia a las mascotas u objetos-, a través de vectores de conexión de diferente diseño (continuo o discontinuo). Esta estrategia gráfica permite al lector generar una suerte de relaciones significativas entre personajes, la cual se antoja

19 Alonzo Reed y Brainerd Kellogg expusieron su sistema en Higher Lessons in English (1877). 

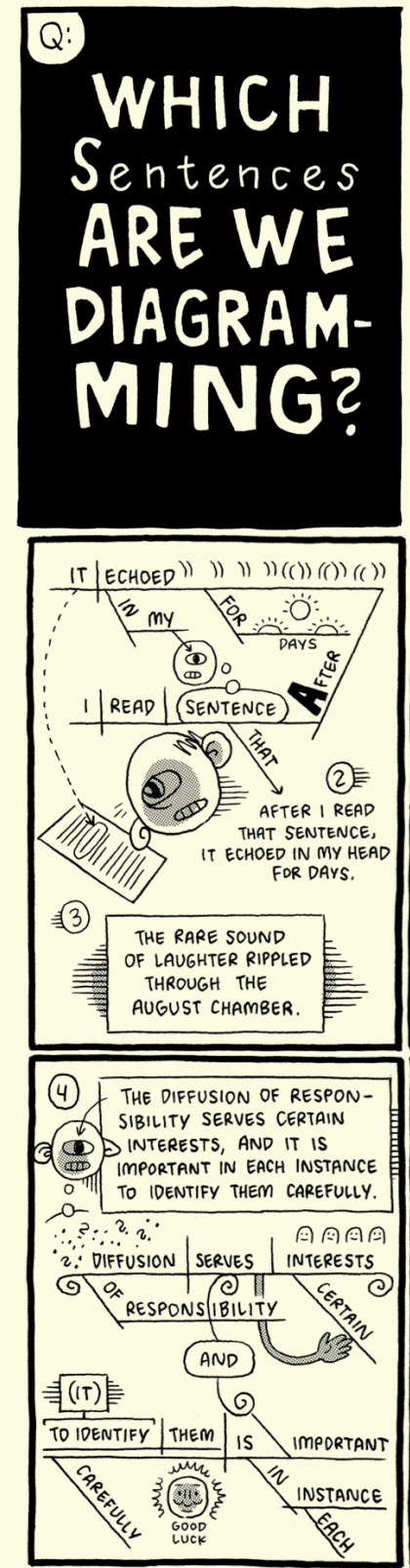
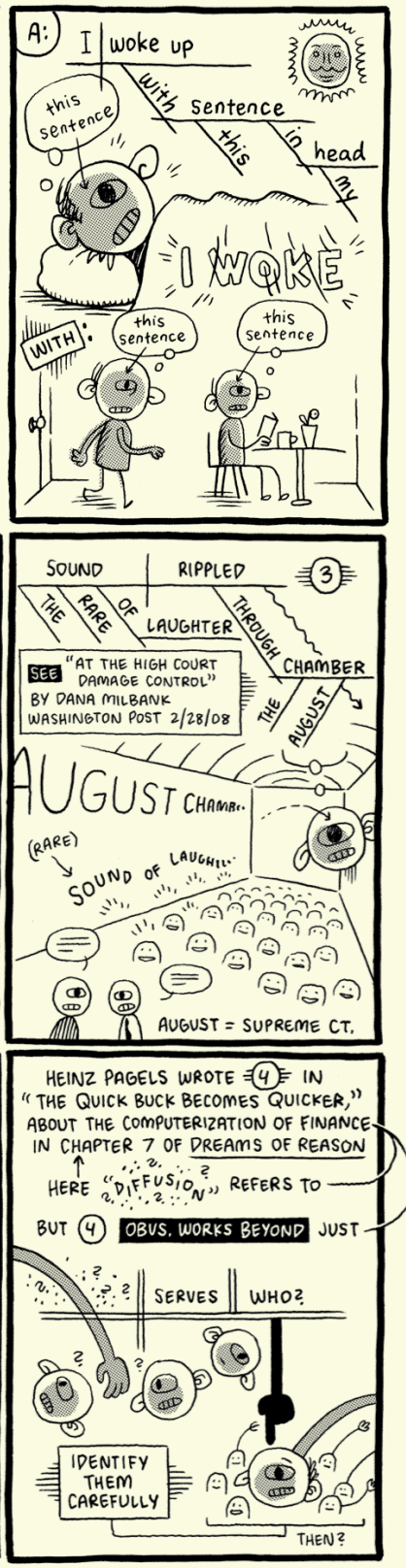
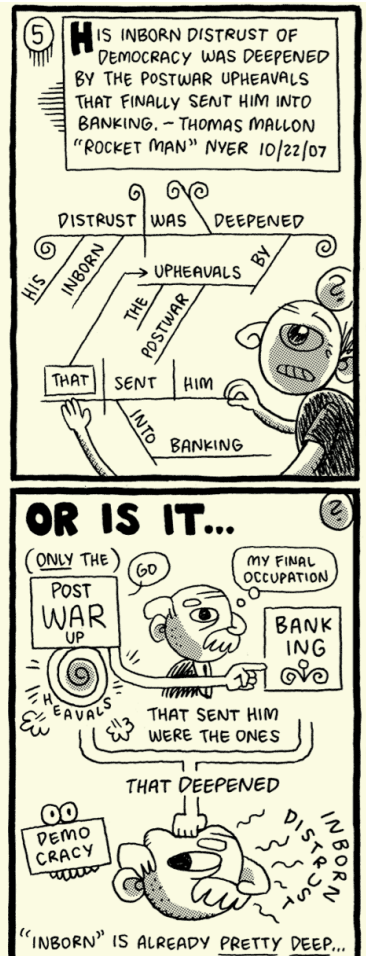

(2.) WHO MAGNUS VON BRAUN, 1. (FATHER OF WERNER VON BRAUN, (2) ROCKET SCIENTIST) "INBORN 1.) IS THIS EVEN POSSIBLE

(2.) "POSTWAR UPHEAVALS"

(1) POST WWI, ROUGH TIMES

(2) "FINALLY SENT HIM INTO ?$$
\text { 1) BANKING" }
$$
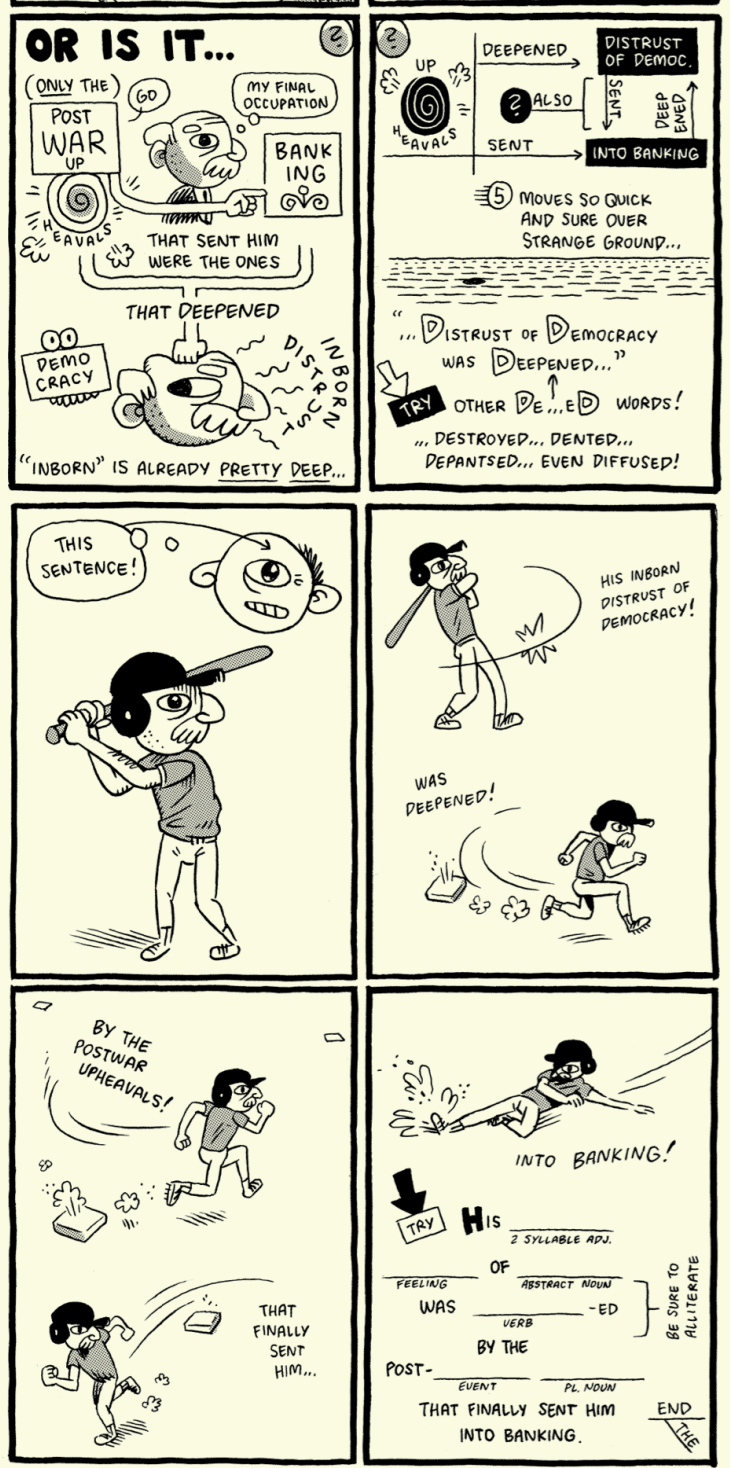

FIG. 2. "Which Sentences Are We Diagramming?". K. Huizenga. Or Else n. ${ }^{\circ}$ 5. Drawn \& Quarterly (2008). http://whatthingsdo.com/comic/which-sentences-are-we-diagramming/

disparatada y humorística como es habitual en las exposiciones de Leon Beyond. La paradoja que anunciábamos resulta de la consideración que adquiere el esquema, debido a la evolución temporal del mismo, que permite visualizar tanto las relaciones como los desarrollos temporales. Sin embargo, dado que en el diseño del mismo la cronología se hace confusa (pues se reseña en el texto la correspondencia en fechas, pero esta no tiene relevancia en el conjunto del diagrama), hemos considerado que domina la característica de relación jerárquica a la de relación temporal, razón por la que lo clasificamos dentro de esta familia. 


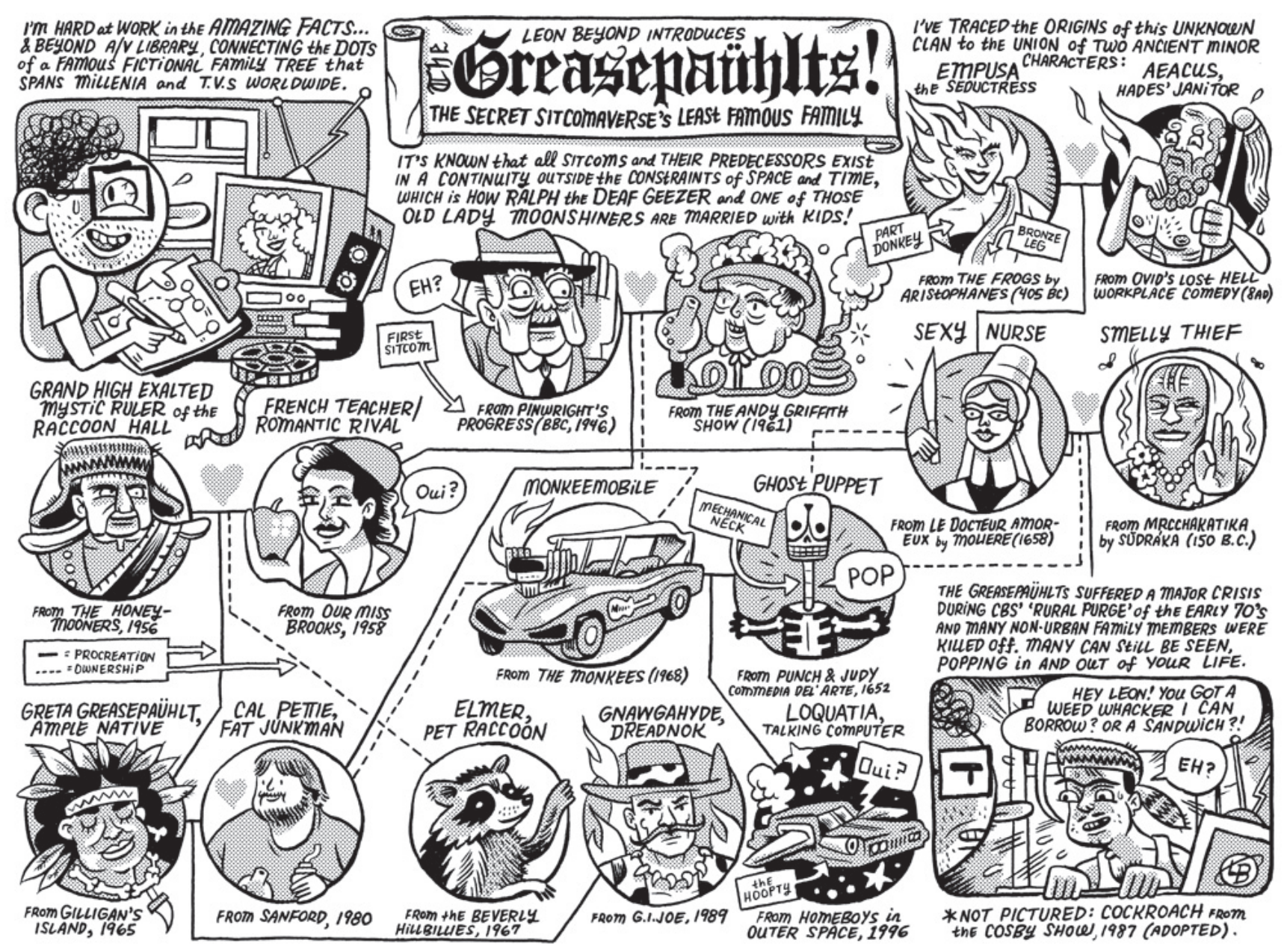

FIG. 3. The Greasepaühlts. Amazing Facts and Beyond with Leon Beyond. D. Zettwoch. Uncivilized Books (2014) http://leonbeyondfacts.blogspot.com.es/2011/11/greasepauhlts.html

\section{c) Datos}

De nuevo este apartado tiene una menor participación en el cómic. Al igual que en el caso anterior tiene extraordinarias excepciones, como la serie The Nightly News de J. Hickmann (2006-07), que muestra de manera paralela a la acción los datos aportados por los medios de comunicación dentro del contexto de la ficción del protagonista. No obstante, el tratamiento gráfico de los datos que hace Hickmann evita su integración tratando de mantenerlos en su dimensión científico analítica, con una estética fiel a la que describíamos como propia del contexto científico y técnico. En cuanto al relato supone un complemento de la historia más que un elemento determinante. Esta postura es sacrificada por D. Zettwoch al acercarse a la cuantificación de una manera más relajada, implicando al territorio del dibujo del cómic en las gráficas de datos y convirtiéndolas en protagonistas de sus narraciones.

Con la estructura ¿sabías qué? mantenida en toda la serie, el pseudointelectual Leon Beyond nos explica, a petición de unos lectores, los promedios de altura del ser humano (FIG. 4). Basándose en las fuentes de su investigación ubica a estos lectores y su desviación del estándar haciendo especial hincapié en el porcentaje de población que es más bajo que cada uno de ellos. Lejos de simplificar su exposición a un frío diagrama de curva de Gauss donde ubica a los remitentes, se explaya gráficamente en la comparativa de los lectores frente a personajes populares combinados con animales extintos, fábulas, mitos y personajes de cuentos, exponiendo las alturas relativas de todos ellos, como si de un 


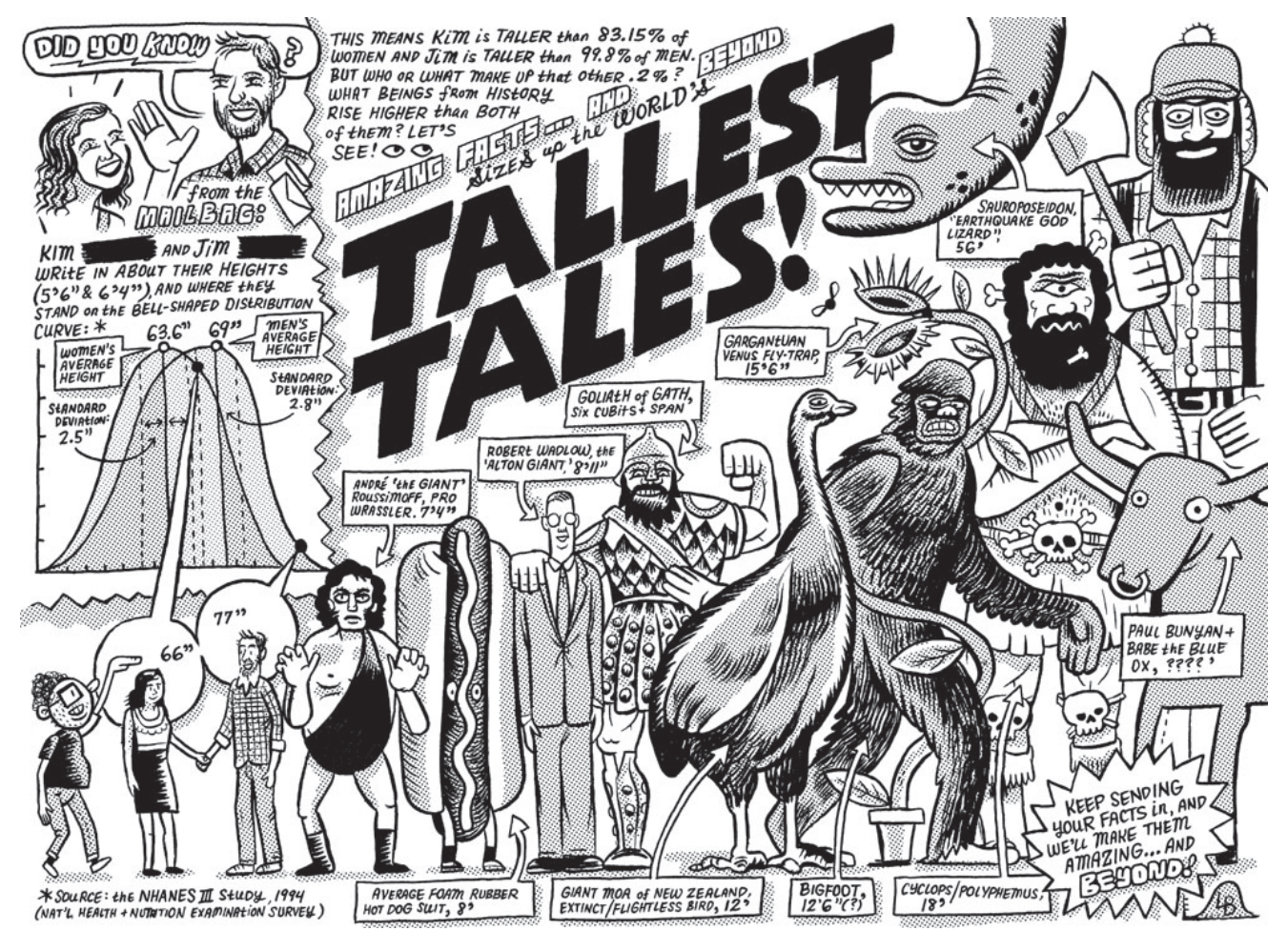

FIG. 4. Tallest tales!. Amazing Facts and Beyond with Leon Beyond. D. Zettwoch. Uncivilized Books (2014) http://leonbeyondfacts.blogspot.com.es/2011/10/tallest-tales.html

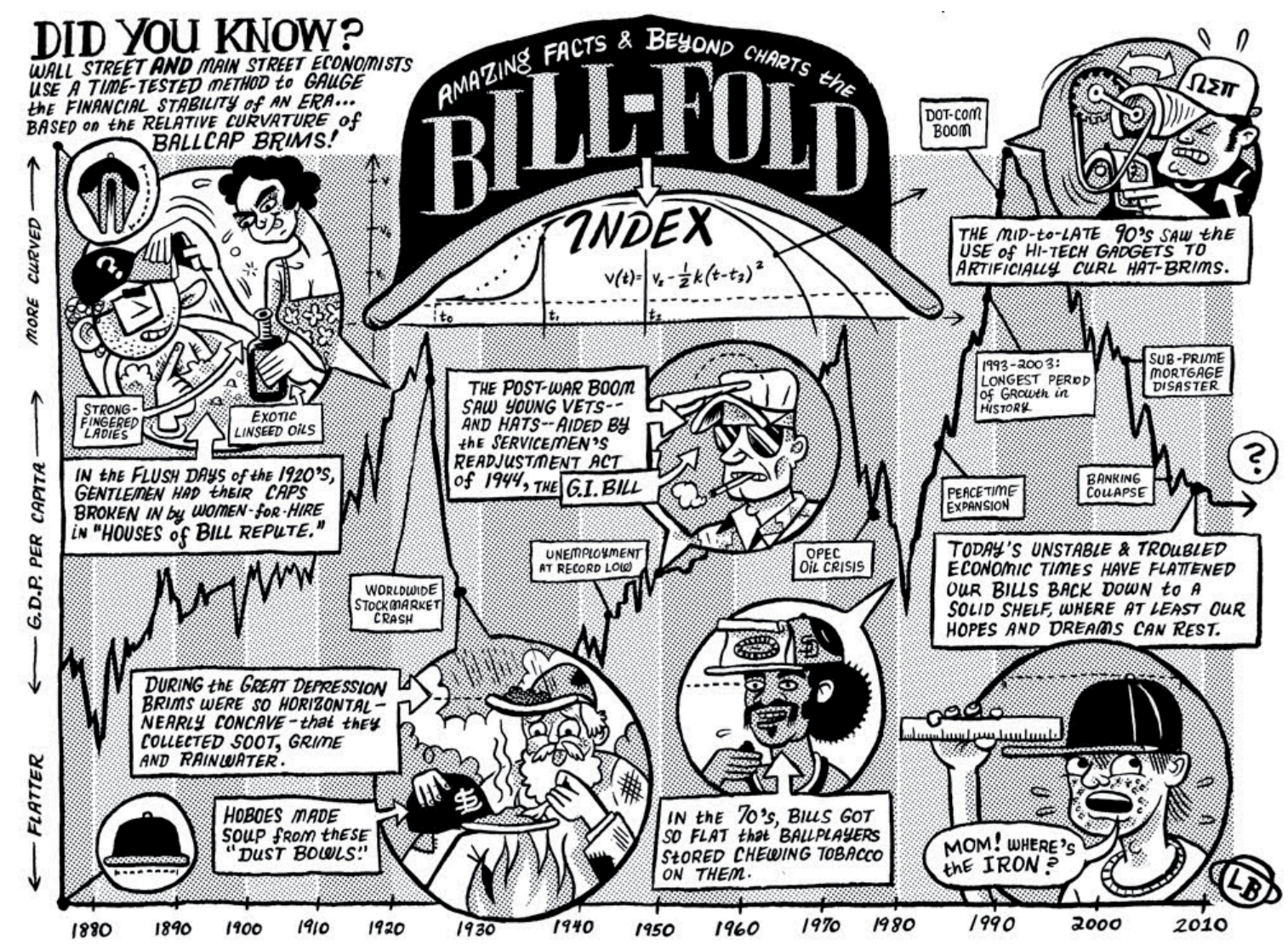

FIG. 5. The Bill-Fold Index. Amazing Facts and Beyond with Leon Beyond. D. Zettwoch. Uncivilized Books (2014) http://leonbeyondfacts.blogspot.com.es/2010/09/bill-fold-index.html 
diagrama de barras se tratara, aunque carece de la fidelidad científica de estos adquiere gran atractivo. Y a pesar de lo anecdótico y humorístico que pueda resultar para el lector, no ceja en su empeño de dar contenido verificable en forma de datos al introducir porcentajes, alturas y datos para mayor aprendizaje del lector.

En la siguiente pieza (FIG 5) Leon Beyond trata de explicar al lector el sistema de medida de la estabilidad financiera de EE. UU.y su relación con la curvatura de la visera de la gorra. Semejante asociación tan solo se puede ofrecer desde una perspectiva humorística, por lo que una gráfica científica como son las habituales del contexto de la bolsa, aunque se mantiene intacta, se impregna de llamadas de texto y de imágenes de ejemplo, en donde radican la explicaciones de la relación economía y estética. El diagrama de fiebre, permite comparar las variaciones de la bolsa americana durante algo más de cien años, el lector puede visualizar globalmente las idas y venidas de la economía, a la par que señala los hitos más relevantes que tuvieron lugar en esta cronología y la vinculación con la forma a la moda de llevar la visera. Su lectura implica una consideración temporal, una cronología, por lo tanto se trata de una construcción esquemática híbrida. Como advertíamos en la descripción de este grupo, los datos son especialmente propensos a ser presentados relacionados con estados o, como en este caso, combinado con un desarrollo temporal.

\section{D) Desarkollos EN El Tiempo}

Tal y como anunciábamos con anterioridad, esta familia de esquemas abraza las formas secuenciales de las instrucciones gráficas, aquellas que nos sirven para el aprendizaje de procedimientos de uso y montaje de los aparatos tecnológicos o de casi cualquier producto manufacturado, así como en las tarjetas de seguridad aérea. Dada la proximidad de estas formas esquemática al propio sistema del cómic, preferimos ejemplificar aquellos desarrollos que por su carácter abstracto abran el espectro de las posibilidades de la esquemática.

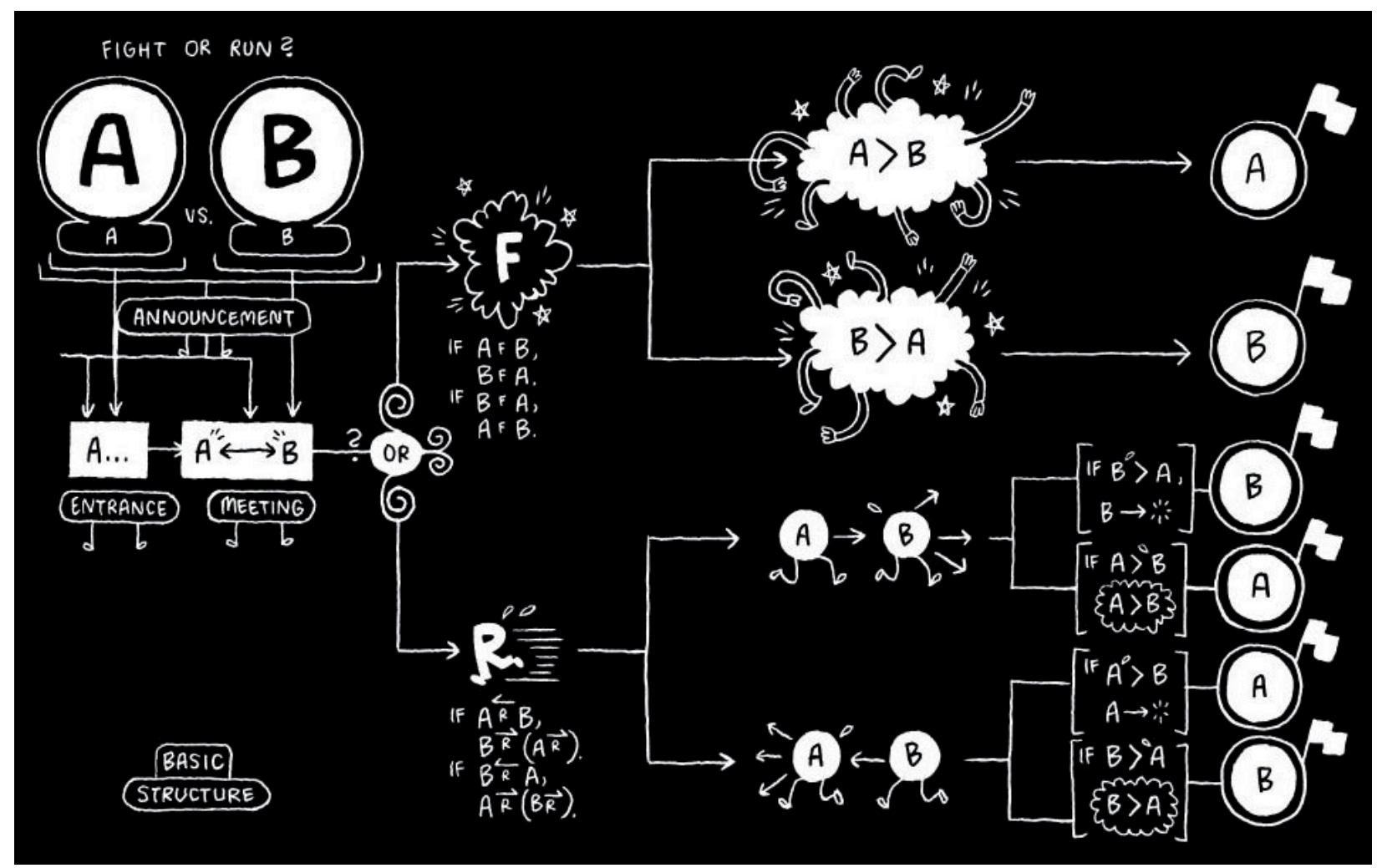

FIG. 6. Fight or Run: Shadow of the Chopper. Kevin Huizenga. Buenaventura Press (2008) http://fightorrun.blogspot.com. es/2010/04/how-to.html 
En este ejemplo Huizenga se sirve de un diagrama de flujo para explicar al lector el funcionamiento básico de la estructura narrativa de la serie Fight or Run, por lo que aquí la esquemática tiene una finalidad expositiva, ya que no forma parte de ningún relato, sino que es contenido extradiegético. Este esquema sirve para que sea el lector el que construya la interpretación de la estructura narrativa de la obra antes de enfrentarse a ella. Esta trama se reduce a una suerte de eventos y acciones que un diagrama en forma de árbol sintetiza de manera efectiva. En esta ocasión la consideración de esquema de desarrollo temporal es clave, en tanto este diagrama explica el desarrollo narrativo y por tanto la evolución del relato. Las secuencias que componen la trama ofrecen alternativas en su desarrollo, pero no en su excusa narrativa, que se resume en una batalla entre adversarios que desemboca en lucha o huida. Se organiza a partir de un anuncio o cabecera de presentación de los rivales, y la posterior entrada en escena y encuentro de los mismos. Esta presentación se repite de manera sistemática, pero las posibilidades a partir de aquí se bifurcan en las dos rutinas que dan nombre al cómic. El desenlace de estos actos of rece al lector un vencedor en todas las combinaciones posibles. Llama poderosamente la atención la gráfica de apariencia matemática que usa el autor para simplificar la explicación de la estructura narrativa propuesta. Podemos comprobar las posibles combinaciones que of rece la trama de este cómic antes del desarrollo del relato, siendo el total de alternativas de seis, con un cincuenta por ciento de probabilidad de éxito para cada uno de los participantes.

La última pieza de la exposición (FIG. 7) resulta la única integrada dentro de una narración, por lo que en este caso se hace evidente la intención narrativa frente a la expositiva, lo cual lo distancia de los ejemplos anteriores al ser un fragmento del propio relato. Aquí el funcionamiento del diagrama de flujo permite presentar desde el inicio alternativas en la dirección de lectura. Los recorridos que Huizenga genera, aunque presentan divergencias, convergen en una única solución. La estructura de conexiones es abstracta, como en el caso anterior, pero el discurso tiene una dependencia mayor del texto. No obstante, ciertos matices se incorporan a través de los gestos de los personajes o los mensaje cifrados en forma de pictogramas que encontramos en el recorrido. Este pequeño relato, aparentemente biográfico, nos traslada a la diatriba del creador al enfrentarse a una creación que etiquetada bajo la denominación "Fashionably Zen" resulta completamente frustrante para el mismo, pero cuyas alternativas resultan igual de deprimentes y conducen a un único final, la miseria económica y laboral del dibujante. Sin embargo, ironiza el autor, al menos le permitirá tener tiempo para dibujar cómics.

La estructura de diagrama de flujo es una de las más interesantes que ofrece la esquemática al cómic debido a que su estructura de relaciones es casual, en analogía a la condición metonímica habitual del cómic. La notable capacidad sintética de la esquemática nos of rece una suerte de posibilidades que podemos examinar conjuntamente, desde su totalidad. Su condición vectorial permite recorridos alternativos que convergen o divergen, ampliando la casuística de la causalidad y pudiendo enriquecer la densidad comunicativa del cómic.

\section{Conclusiones}

Como hemos podido observar en estos ejemplos de Huizenga y Zettwoch se exploran de manera homóloga el cómic de ficción y el cómic de divulgación con la misma capacidad para la traslación de códigos gráficos de territorios a priori ajenos. Los mapas, los esquemas de relaciones y estructuras, los datos y los desarrollos en el tiempo aparecen en sus respectivas obras como elementos determinantes, a menudo protagonistas que organizan el discurso. Hemos advertido que en estas páginas analizadas se presentan de forma alterna las exposiciones y las narraciones, lo cual nos ofrece un panorama donde la narración puede ser explícita o llegar al lector a través de la exploración e interpretación de los códigos como si de un "objeto de trabajo" se tratara.

Señalaremos que la complejidad de los códigos esquemáticos, aquellos que integran mayor densidad, correlaciones y abstracción informativa requieren mayor tiempo para su interpretación. Esta tendencia, 


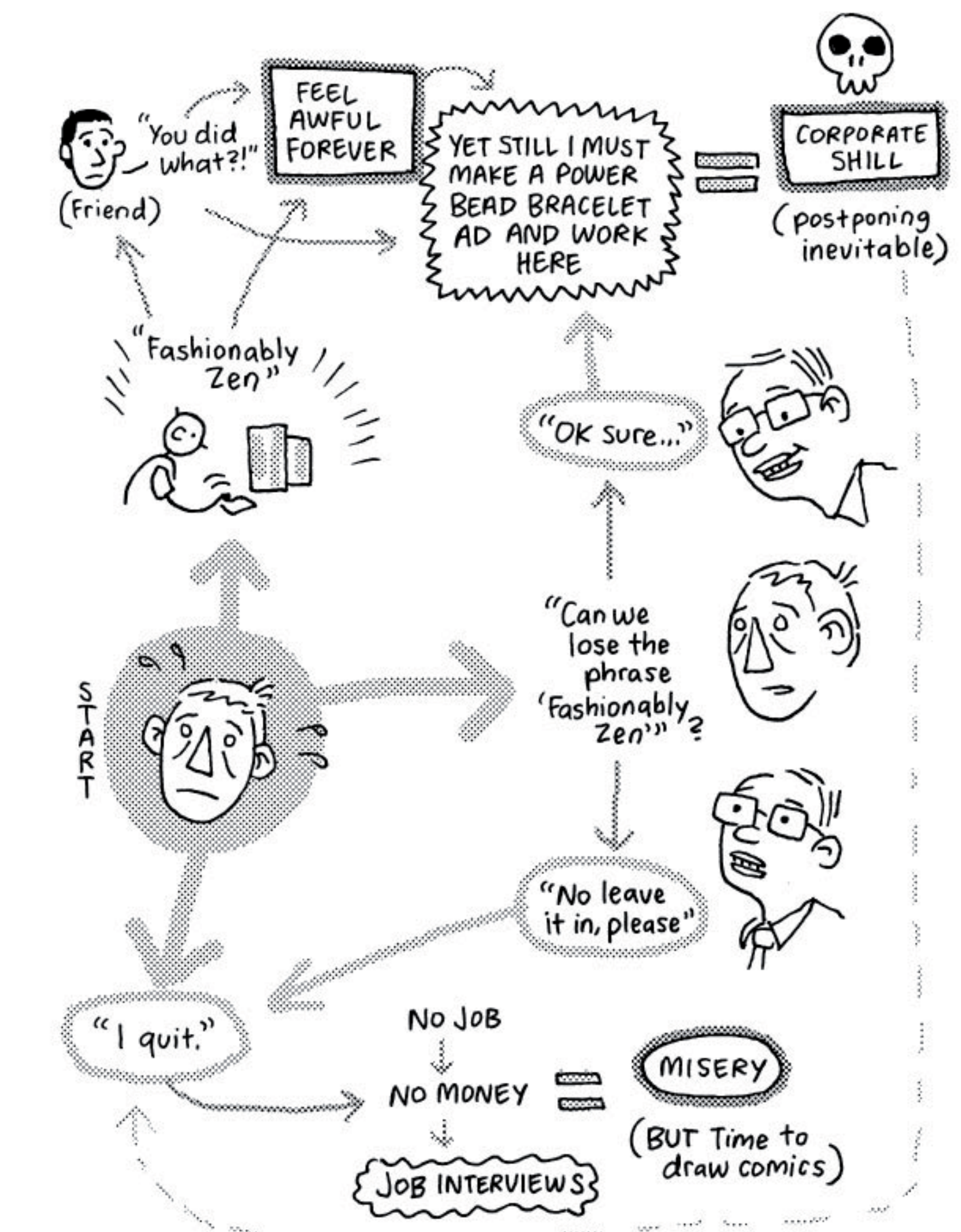


que podría considerarse un obstáculo para la fluidez de la lectura habitual del cómic, aquí la proponemos como otra vía de generar una narrativa. Lejos de considerarla un impedimento se propone como estrategia dirigida a aumentar la riqueza comunicativa, aprovechando el compromiso activo del lector que se ve seducido por una poliédrica narración de la realidad.

Las incorporaciones y traslaciones de los recursos de la esquemática en el cómic se deben a la polivalencia de estos autores, que trabajan desde planteamientos interdisciplinares y que no son ajenos a la formas de comunicación específicas de otras profesiones de la comunicación visual.

Localizados los ejemplos de las familias de la esquemática propuestas, a la pregunta de si hubiera existido una solución más eficaz para narrar los aspectos expuestos por las formas esquemáticas, la respuesta nos ofrece una evidencia notable de la capacidad de este lenguaje para visualizar aquellas realidades no accesibles a la vista y, en consecuencia, no pudiendo ser narrado de forma tan efectiva según el sistema convencional del cómic basado en la "solidaridad icónica" ${ }^{20}$ Estos casos nos han permitido conocer un recorrido y su ruta en un mapa; han descompuesto y analizado un discurso textual y han puesto de relevancia de manera sintética una relación de personajes de manera optimizada; han posibilitado cuantificar y comparar en relación a la media humana las alturas de personajes singulares y nos han mostrado la evolución de la bolsa a lo largo de más de un siglo en una estructura simple; por último, nos han sintetizado la estructura básica narrativa de toda una serie, así como han hecho visibles los recorridos vitales que implican las decisiones de un creador.

Podemos concluir, a través de este análisis, que las posibilidades que ofrece la esquemática al cómic según hemos observado en la obra de Huizenga y Zettwoch, están directamente relacionadas con la amplificación de las posibilidades de comunicación, al incorporar dimensiones y/o puntos de vista de la realidad anteriormente no expuestas. Se ha comprobado la facilidad para generar claridad y comunicación en torno a las relaciones y conexiones, además de abrir a las posibilidades al aumento de la densidad informativa, la solidaridad no icónica, la síntesis de información y al análisis de datos; brindando al cómic la oportunidad para ampliar sus fronteras a través de la exploración de este lenguaje. Las consecuencias que también advertimos y en las que coincidimos con $\mathrm{Cates}^{21}$ es que estas experiencias conllevan una ruptura con la lectura fluida habitual entre viñetas al proponer al lector una pausa para la exploración. Sin embargo, construir la narración a través de la exploración no deja de ser más que otra forma de lectura, más exigente con el lector, pero cuya recompensa consideramos que merece poner en práctica.

20 Groensteen, T. The System of Comics. Jackson, University Press of Mississippi, 2007, pp. 17-20.

${ }^{21}$ Cates, I. “Comics and the Grammar of Diagrams”, en BAll, D. (ed.). The comics of Chris Ware: drawing is a way of thinking. Jackson, University Press of Mississippi, 2010, pp. 90-102. 


\section{Bibliografía:}

BARbieri, D. Los lenguaje del cómic. Barcelona, Paidós, 1993.

Bender, J. y Marrinan, M. The Culture of Diagram. Stanford, Calif., Stanford University Press, 2010.

CAIro, A. El alfabeto funcional. Madrid, Alamut, 2011.

Cates, I. "Comics and the Grammar of Diagrams", en BAll, D. (ed.). The comics of Chris Ware: drawing is a way of thinking. Jackson, University Press of Mississippi, 2010. pp. 90-102.

Costa, J. La esquemática: visualizar la información. Barcelona, Paidós, 1998.

Franchi, F. “Literatura gráfica”, en VVAA. Malofiej 18. Pamplona, Index Book, 2011, pp. 50-57.

Froenlich, C. "Gotham City and Metropolis can keep their clichés- St. Louis is flying high in the edgy world of alternative comics”, entrevista con Kevin Huizenga, en St. Louis Magazine. 2007. http://www.stlmag.com/ Cartoon-Heroes/ (consultado el 8 de mayo de 2013).

García, S. La novela gráfica. Bilbao, Astiberri, 2010.

García Sánchez, S. Anatomía de una historieta. Madrid, Sinsentido, 2004.

Groensteen, T. The System of Comics. Jackson, University Press of Mississippi, 2007.

McCloud, S. Understanding comics: the invisible art. New York, Harper Perennial, 1994.

Melero, X. "El cómic como medio periodístico", Revista Eutopias. Vol. 1-2. (2011). http://www.eu-topias.org/ articulo.php?ref page=92 (consultado el 27 de marzo de 2013)

Rall, H. "Comics and Interactive information Graphics", en Interaktive Infografiken, editores. Berlin, Springer Vieweg, 2013, pg. 175-215.

Tufte, E. Envisioning information. Cheshire, Conn., Graphics Press, 1995. 


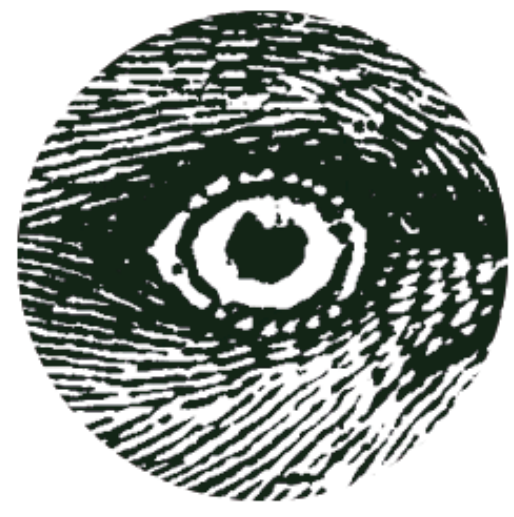

